\title{
Altération fonctionnelle par irradiation gamma (1, 2 et 3 Gy) du fibroblaste de derme humain, au cours du vieillissement cellulaire in vitro (Collagène, fibronectine, métalloprotéinase, métallo-endopeptidase)
}

\author{
V. RENAUD-SALIS, L. SABATIER ${ }^{*}$, R. MASSE*, C. LAFUMA \\ Laboratoire de biologie du tissu conjonctif, CNRS 1460, Créteil ; \\ *Commissariat à l'énergie atomique, DSV/DPTE, \\ BP 6, 92265 Fontenay-aux-Roses Cedex
}

Afin de mieux appréhender les mécanismes d'induction de la fibrose post-radique, nous avons étudié les capacités de prolifération ainsi que la réponse fonctionnelle et le caryotype de fibroblastes issus de derme humain et irradiés, par comparaison aux mêmes fibroblastes non irradiés.

Dans ce but et afin de nous extraire de tout contexte environnemental tissulaire inflammatoire ou lésionnel et de tester l'effet seul de l'irradiation, nous avons utilisé le modèle de Hayflick et choisi de délivrer aux fibroblastes jeunes à confluence, des doses d'irradiation relativement modérées $(1,2$ et $3 \mathrm{~Gy})$. Nous avons ensuite, tout au long des sous-cultures jusqu'au stade ultime de la sénescence fibroblastique, étudié l'expression du collagène et de la fibronectine, principales macromolécules constitutives de la matrice extracellulaire, ainsi que l'activité des métalloprotéases impliquées dans la dégradation et le renouvellement de cette matrice.

Les résultats montrent que l'irradiation in vitro des fibroblastes favorise l'établissement progressif et tardif en phase de sénescence d'un phénotype à caractère "fibrosant" se rapprochant de celui exprimé par les fibroblastes issus de fibrose porcine sous-cutanée post-radique. L'intensité de cette réponse tardive fibroblastique à "caractère fibrosant" paraît dépendante de la dose d'irradiation initialement délivrée et paraît corrélée au taux d'anomalies chromosomiques dépourvues de caractère clonal et observées dès le début.

Enfin, cette réponse peut être partiellement potentialisée par une cytokine telle que le TGFB et suggère que l'irradiation puisse également sensibiliser les fibroblastes à l'action d'autres cytokines susceptibles de moduler l'expression d'un phénotype fibrosant. 